\title{
Protective measures undertaken during chest tube thoracostomy in COVID-19 outbreak
}

\author{
İsmail Ertuğrul Gedik ${ }^{1}$ (1) - Timuçin Alar ${ }^{1}$
}

Received: 19 September 2020 / Revised: 29 October 2020 / Accepted: 2 November 2020 / Published online: 24 November 2020

(C) Indian Association of Cardiovascular-Thoracic Surgeons 2020

\begin{abstract}
Background Coronavirus disease 2019 (COVID-19) is a newly emerging infectious disease that was first reported in China and has become a worldwide pandemic. Many surgical procedures are continuing to be performed during this state of pandemic as is thoracic surgery. We present six cases of tube thoracostomy in COVID-19 patients and the modifications to the routine surgical technique.

Methods We serially attached two closed underwater drainage systems (CUDS) together and added a high-efficiency particulate air (HEPA) filter to the port of the second CUDS, because the intrapleural air, which passes through the CUDS into the air in intensive care unit (ICU), may contain high concentrations of 2019 novel coronavirus (2019$\mathrm{nCoV}$ ). Second, we attached the chest drain to the first CUDS in order to prevent the spread of virus during the placement of drain into the pleural cavity. Third, just before opening the parietal pleura, ventilation was put on standby mode and the endotracheal tube was clamped to prevent viral dissemination to the environment. Fourth, we covered the incision with a gauze sponge soaked with sterile saline solution during pleural entry, to prevent viral dissemination into the environment.

Results There were a total of six patients enrolled in our study. All these patients were diagnosed with COVID-19. The surgical indication for the chest tube thoracostomy was tension pneumothorax in all six patents. All patients had lung expansion defects and subcutaneous emphysema after intervention. Unfortunately, all of them succumbed to COVID-19, despite best available treatment. There was no COVID-19 infection reported in the healthcare professionals during this study.

Conclusions Thoracic surgical procedures may cause dissemination of high amounts of $2019-\mathrm{nCoV}$ in the environment and thus are perhaps the most dangerous surgeries to perform. Variations in the thoracic surgical techniques are necessary in order to protect the healthcare providers from COVID-19.
\end{abstract}

Keywords Tube thoracostomy $\cdot$ COVID-19 $\cdot$ Protective measures $\cdot$ Tension pneumothorax

\section{Introduction}

Coronavirus disease 2019 (COVID-19) is a newly emerging infectious disease that was first reported in China and has become a worldwide pandemic [1]. Many surgical procedures are continuing to be performed during this state of pandemic, as is thoracic surgery [1]. Here we present a case series about our experience in chest tube/

İsmail Ertuğrul Gedik

ertugrulgedik@gmail.com

1 Department of Thoracic Surgery, Çanakkale Onsekiz Mart University Faculty of Medicine, Çanakkale, Turkey catheter thoracostomy and the special procedures performed in patients who were diagnosed with COVID-19 to prevent the spread of infection to the healthcare providers.

\section{Patients and methods}

This study was conducted in Çanakkale Onsekiz Mart University Faculty of Medicine Hospital between 1 April 2020 and 15 April 2020. Patients who were treated in the intensive care unit (ICU) with the diagnosis of COVID-19 and were intervened by the Department of Thoracic Surgery were enrolled in this study. 


\section{Surgical method}

Before performing these interventions, thoracic surgical team wore protective equipment, including goggles, sterile surgical gloves, protective full body suits, and European Norm-149 (EN-149) standard high particle filter masks.

\section{Chest tube thoracostomy}

Chest tube thoracostomy was performed in cases with pneumothorax. Informed consent was obtained from the first-degree relatives of the patients, because all patients were on mechanical ventilator (MV) and their Glasgow Coma Scale (GCS) was 3. Before the start of the procedure, surgical material and equipment were prepared in a sterile environment. We used two closed underwater drainage systems (CUDS) serially attached together. The line of the second CUDS was divided with a sterile scalpel and the divided line was attached to the first CUDS's port. A high-efficiency particulate air (HEPA) filter with the viral filtration efficiency (VFE) rating of $99.999 \%$ according to European Norm 1822-1:2009 (EN 18221:2009) standards was used to filter the air coming out of the port of the second CUDS (Fig. 1). After the preparation of the CUDS, we attached the chest drain to the first CUDS (Fig. 2). Skin asepsis was ensured with $10 \%$ solution of povidone iodine to the relevant hemithorax. Local anesthesia was not required since all patients were unconscious. A skin incision of approximately $15 \mathrm{~mm}$ width at the intersection of fifth intercostal space and the mid-axillary line was performed with a number 20 scalpel. A subcutaneous tunnel was established by dissecting subcutaneous tissues and intercostal muscles using a curved Metzenbaum scissors, at the superior border of

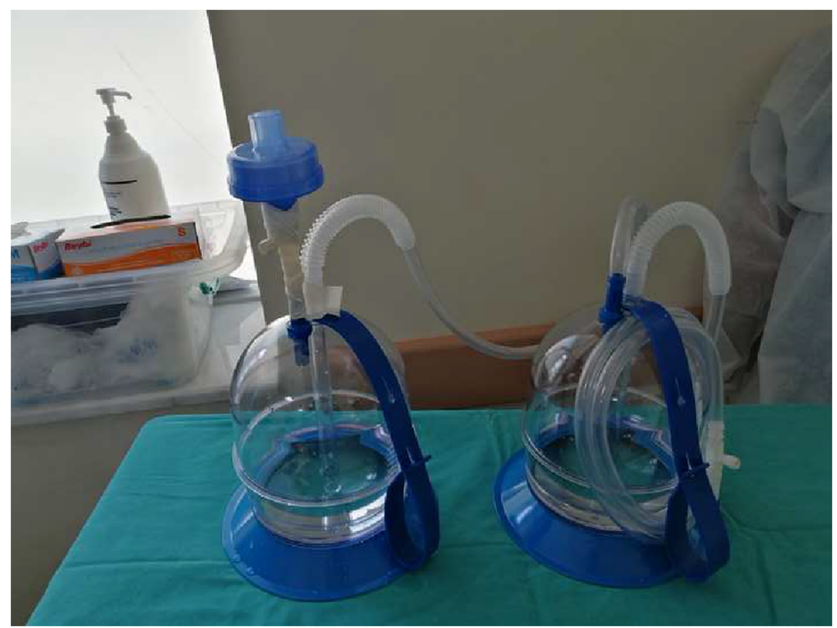

Fig. 1 Two closed underwater drainage systems serially attached together with a high-efficiency particulate air (HEPA) filter

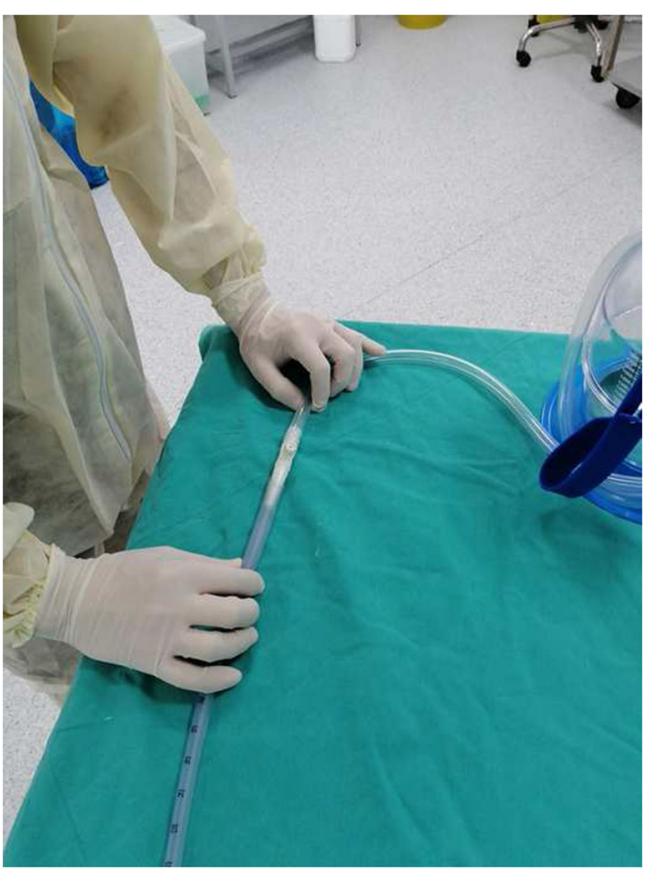

Fig. 2 Chest drain is attached to the first closed underwater drainage system

the sixth rib. After the tunnel was created, drain fixation suture was placed into both corners of the skin incision with atraumatic sharp United States Pharmacopeia (USP) number 1 silk suture, and a U-shaped suture was put into the middle part of the incision with the same suture material. This U-shaped suture was used to close the incision during drain removal. Just before opening the parietal pleura, MV was put on standby mode and the endotracheal tube was clamped to prevent viral dissemination in the environment. Then, the pleura was carefully opened using Metzenbaum scissors, with a gauze sponge soaked with sterile saline solution wrapped around the scissors and covering the incision during pleural entry, to prevent viral dissemination from the pleural cavity. With the help of a clamp, a 32-French (Fr) rigid chest drain was placed in the thoracic cavity and advanced towards the apex. After monitoring the oscillation of the water column and air drainage, the drain was fixed to the skin of the patient using the fixation sutures. The U-shaped suture was left unknotted and wrapped around the drain. The process was terminated by dressing the wound.

Twenty-four hours after the procedure, patients were reevaluated with a chest $\mathrm{x}$-ray. The position of the drain in the thorax and the lung expansion in the thoracic cavity was monitored. In daily follow-up, the condition of the dressing, the presence of drainage, and oscillation of the water column were evaluated. Complete expansion of the lung and absence of air drainage continuously for $48 \mathrm{~h}$ were considered the criteria for the removal of the chest drain. 


\section{Results}

There were a total of six patients enrolled in our study. All these patients were diagnosed with COVID-19 using clinical and radiological data with or without reverse transcriptase polymerase chain reaction (RT-PCR) from the sample obtained by endotracheal aspiration. The surgical indication for the chest tube thoracostomy was tension pneumothorax in all six patients. Age of these patients ranged from 44 to 84 years and all of them were ventilated at pressure-controlled ventilation (PCV) mode. The main characteristic of all these six pneumo-thoraces was that the collapse of the pulmonary parenchyma was minimal, but there were other signs indicating tension pneumothorax. These were bradycardia, hypotension, contralateral shift of the mediastinum on chest $\mathrm{x}$-ray, and sudden high-pressure air coming out of the surgical site during entry into the pleural cavity (Fig.3). There were other common findings in these patients after chest tube thoracostomy in form of lung expansion defects and subcutaneous emphysema after intervention.

Unfortunately, all six patients succumbed to COVID-19. However, there was no COVID-19 infection reported in the healthcare professionals during this study.

\section{Discussion}

Coronavirus virions are spherical with diameters of approximately $125 \mathrm{~nm}$. The defining feature of coronaviruses is the club-shaped spike projections emanating from the surface of the virion, which give them the appearance of a solar corona, prompting the name coronaviruses [2].

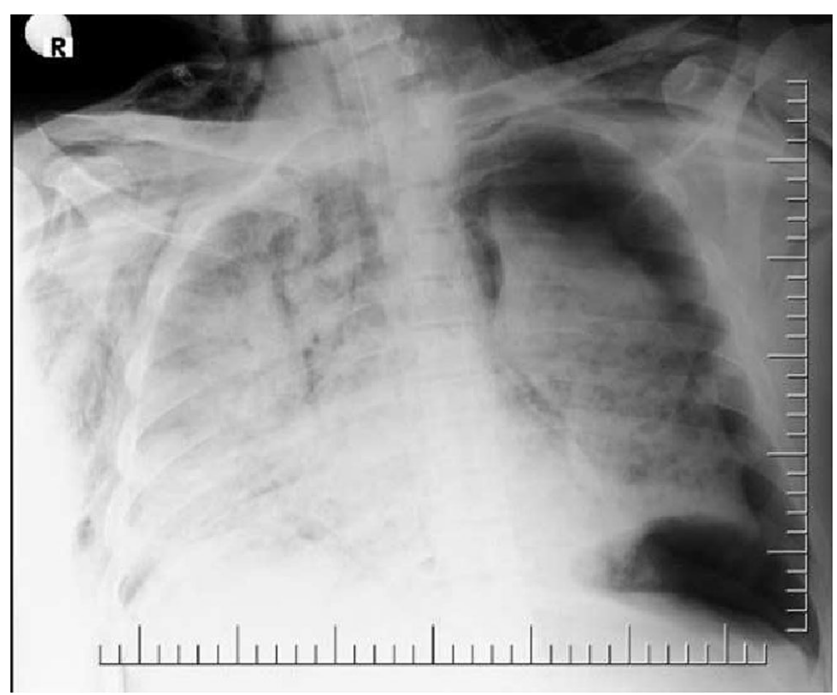

Fig. 3 Chest x-ray depicting left tension pneumothorax
COVID-19 outbreak issues a challenging working environment for physicians and healthcare providers. There are numerous reports of healthcare workers getting infected and dying throughout the world. As of early March 2020, 3300 healthcare workers had been infected in China and about $20 \%$ of healthcare providers had been infected in Italy [3]. These reports came despite strict protective measures being taken such as wearing protective equipment. We too used protective equipment (goggles, sterile surgical gloves, protective full body suits, EN-149 standard high particle filter masks) before performing tube thoracostomies, since all patients who were enrolled in this study were confirmed COVID-19 cases. All thoracic surgical procedures, including tube thoracostomy, carry a higher risk of contracting and disseminating disease than surgeries on other organs, because the lung has the highest concentration of 2019 novel coronavirus (2019-nCoV) [4]. We have modified our regular tube thoracostomy method in four steps in order to prevent the spread of the virus into the environment and thus protecting the ICU personnel. First, we serially attached two closed CUDS together and added a HEPA filter with the VFE rating of $99.999 \%$ according to EN 1822-1:2009 standards to filter the air coming out of the port of the second CUDS, because the intrapleural air draining through the CUDS into the air in ICU may contain high concentrations of 2019-nCoV. HEPA filters are manufactured by pleating multiple layers of microfiber glass, or other fibrous media, as randomly arranged fibers, with diameters ranging from 2 to $500 \mathrm{~nm}$ and are efficient in filtering viral particles [5]. Second, we attached the chest drain to the first CUDS to prevent the spread of virus during the placement of drain into the pleural cavity. Third, just before opening the parietal pleura, MV was put on standby mode and the endotracheal tube was clamped to prevent viral dissemination to the environment. Fourth, we covered the incision with a gauze sponge soaked with sterile saline solution during pleural entry to prevent viral dissemination into the environment. We propose the use of this method for chest tube thoracostomies as a protective measure for the healthcare professionals.

All patients in our study were tension pneumothorax. We observed severe, sudden respiratory distress, despite limited parenchymal collapse, bradycardia, hypotension, and contralateral shift of the mediastinum on chest $\mathrm{x}$ ray. This was confirmed by high-pressure air coming out of the surgical site during entry into the pleural cavity. In addition to these, sudden recovery from bradycardia, hypotension, and respiratory distress to some extent was also suggestive of tension pneumothorax. Tension pneumothorax, despite limited parenchymal collapse, can be expected in patients with COVID-19, because it has been reported that COVID-19 causes interstitial pneumonia with 
fibroblast proliferation $[6,7]$. We believe that like in other cases of interstitial lung diseases, COVID-19 causes a significant decrease in lung elastance and prevents further collapse during pneumothorax. Tension pneumothorax might occur because of this relative rigidity of the pulmonary parenchyma, compared to the mediastinal and other tissues surrounding the lung. This rigidity of the pulmonary parenchyma may also be the cause of pneumothorax in a similar pathogenesis to interstitial lung diseases, which are known to develop pneumothorax [8].

There are numerous case reports and review of literature about pneumothorax occurring in COVID-19 patients with variable outcomes. Though several patients have been reported to have favorable outcomes, mortality rates were significantly high [9]. Since all patients in our limited experience have died, it is reasonable to say that pneumothorax is an important additional cause of mortality, especially in COVID-19 patients.

\section{Limitations}

Small observational cohort is a major limitation. We believe our proposals for the prevention of viral spread should be tested in a larger study. We would stand vindicated if the sterile saline in the CUDS and HEPA filter were tested and found contaminated with 2019-nCoV.

\section{Conclusions}

COVID-19 is a dangerous infectious disease that threatens healthcare professionals. Thoracic surgical procedures may cause the spread of high amounts of 2019-nCoV from lung parenchyma, air in the pleural cavity, or subcutaneous emphysema to the environment. In COVID-19 patients, pneumothorax is an important additional cause of mortality. It should be kept in mind that in tension pneumothorax in COVID-19 patients, and in fact, as in all tension pneumothorax cases, clinical picture is the most important diagnostic tool. We also believe that just as the humanity has been caught off guard by this COVID-19 pandemic, it is highly probable that new types of infectious pandemics may occur in the future and it is of paramount importance to innovate methods that increase the safety of the healthcare providers.

Funding Authors received no financial support for this article.

\section{Compliance with ethical standards}

Conflict of interest The authors declare that they have no conflict of interest.

Ethical approval This study was approved by The Scientific Research Platform of the Ministry of Health of the Republic of Turkey (2020-0616T12_26_37) and The Clinical Research Ethics Committee of the Çanakkale Onsekiz Mart University Faculty of Medicine (2020-09).

Human and animal rights The procedures followed were in accordance with the ethical standards of the responsible committee on human experimentation (institutional and national) in our study.

Informed consent A verbal and written informed consent was obtained from all of the patients' first-degree relatives.

\section{References}

1. Cai Y, Hao Z, Gao Y, et al. COVID-19 in the perioperative period of lung resection: a brief report from a single thoracic surgery department in Wuhan. China J Thorac Oncol. 2020;15:1065-72.

2. Fehr AR, Perlman S. Coronaviruses: an overview of their replication and pathogenesis. Methods Mol Biol. 2015;1282:1-23.

3. The Lancet. COVID-19: protecting health-care workers. Lancet. 2020;395(10228):922. https://doi.org/10.1016/S0140-6736(20) 30644-9.

4. Wu YC, Chen CS, Chan YJ. The outbreak of COVID-19: an overview. J Chin Med Assoc. 2020;83:217-20.

5. Christopherson DA, Yao WC, Lu M, Vijayakumar R, Sedaghat AR. High-efficiency particulate air filters in the era of COVID-19: function and efficacy. Otolaryngol Head Neck Surg. 2020. https://doi. org/10.1177/0194599820941838.

6. Guan WJ, Liang WH, Zhao Y, et al. Comorbidity and its impact on 1590 patients with Covid-19 in China: a nationwide analysis. Eur Respir J. 2020;55:2000547.

7. Tian $\mathrm{S}, \mathrm{Hu} \mathrm{W}, \mathrm{Niu} \mathrm{L}$, Liu H, Xu H, Xiao SY. Pulmonary pathology of early-phase 2019 novel coronavirus (COVID-19) pneumonia in two patients with lung cancer. J Thorac Oncol. 2020;15:700-4.

8. King TE. Approach to the adult with interstitial lung disease: clinical evaluation. In: Post T, ed. UpToDate. Waltham, MA.: UpToDate; 2019. https://www.uptodate.com/contents/approach-to-the-adultwith-interstitial-lung-disease-clinical-evaluation. Accessed on: 27/ 07/2020.

9. Quincho-Lopez A, Quincho-Lopez DL, Hurtado-Medina FD. Case report: pneumothorax and pneumomediastinum as uncommon complications of COVID-19 pneumonia-literature review. Am J Trop Med Hyg. 2020;103:1170-6.

Publisher's note Springer Nature remains neutral with regard to jurisdictional claims in published maps and institutional affiliations. 